\title{
Competencias emocionales en jóvenes con factores de riesgo psicosocial
}

\author{
Emotional competences in young people with psychosocial risk factors
}

\section{Rosalva Teyes}

rteyes@hotmail.com

Código ORCID: 0000-0002-2299-2552

Universidad del Zulia, Venezuela

\author{
Deleana Rincón Fuenmayor \\ deleanarincon@gmail.com \\ Código ORCID: 0000-0001-8438-0044 \\ Universidad del Zulia, Venezuela
}

\author{
Julianny Ventura \\ julianny.ventura@gmail.com \\ Código ORCID: 0000-0003-2627-1223 \\ Universidad del Zulia, Venezuela
}

Recibido: Abril 2019 / Arbitrado: Mayo 2019 / Publicado: Julio 2019

RESUMEN

Las competencias emocionales nacen de la inteligencia emocional, propuesta en sus comienzos por Salovey y Mayer y popularizada por Goleman con estudios más amplios y reconocidos a nivel internacional. El propósito de esta investigación fue determinar las competencias emocionales presente en los jóvenes del Centro de Capacitación San Juan Bosco, Punto Fijo, Venezuela. Se fundamentó en el enfoque teórico de Bisquerra (2003). Se evaluaron 59 participantes de los diferentes talleres. La medición se hizo con un cuestionario de competencias emocionales; con una confiabilidad alfa de Cronbach de 995.Investigación descriptiva, correlacional de campo. Se concluyó que existe una correlación positiva significativa entre las dimensiones de competencias intrapersonales y competencias interpersonales. Poseen más desarrollada la dimensión interpersonal, que la intrapersonal, por lo que son capaces de establecer diferencias entre los distintos estados de ánimo, intenciones, motivaciones y emociones de otras personas, además de las habilidades para comprender cómo comunicarse adecuadamente.

Palabras clave: Competencias; emociones; inteligencia emocional; adolescentes; jóvenes

\begin{abstract}
Emotional competences are born from emotional intelligence, first proposed by Salovey and Mayer and popularized by Goleman with more extensive and internationally recognized studies. The purpose of this research was to determine the emotional competencies present in the youth of the San Training Center Juan Bosco, Punto Fijo, Venezuela. It was based on the theoretical approach of Bisquerra (2003). 59 participants from the different workshops were evaluated. The measurement was made with an emotional skills questionnaire; with an alpha reliability of Cronbach of 995. Descriptive, correlational field research. It was concluded that there is a significant positive correlation between the dimensions of intrapersonal competences and interpersonal competences. The interpersonal dimension is more developed than the intrapersonal dimension, so they are able to establish differences between the different states of mind, intentions, motivations and emotions of other people, in addition to the skills to understand how to communicate properly.
\end{abstract}




\section{INTRODUCCIÓN}

Las instituciones del siglo XXI han asumido la necesidad de educar a las personas en los nuevos conocimientos técnicos y teóricos oportunos para sobrevivir en la sociedad de la información y el conocimiento en la que nos hallamos inmersos. Si analizamos los programas de estudios, nos encontramos que más allá de los contenidos específicos informativos, tratan de invertir y mejorar algunos de los aspectos emocionales y sociales de los estudiantes, aunque sin partir en la mayoría de los casos, teórico explícito. No obstante, de forma simultáneamente a la sociedad del conocimiento coexiste la sociedad de riesgo.

La Secretaría Regional de las Naciones Unidas para los Derechos Humanos (2005), refiere sobre los factores de riesgos que incrementan la probabilidad de ocurrencia de la violencia en niños, niñas y adolescentes, señalan que existen factores individuales demográficos (edad, género), biológicos, exposición temprana a la violencia, nivel socioeconómico, educacional, unido a la situación laboral de los padres o de los adolescentes, así como los propios del hogar como el tamaño, densidad, estructura, dinámica, normas, patrones de crianza, historia de violencia familiar, abuso del alcohol y drogas.

La UNICEF (2011) plantea que los adolescentes de los grupos marginados no reciben instrucción secundaria incluso cuando este tipo de educación está disponible. De allí la importancia de brindarles una educación para la vida a fin de darle los conocimientos para mejorar su calidad de vida; podrían beneficiarse de cursos no estructurados o de educación de pares, de cursos de formación profesional y técnica, además de programas flexibles destinados a jóvenes que han tenido que interrumpir su preparación académica o profesional debido a condiciones socioeconómicas desfavorables.

En consecuencia, es apremiante la incorporación de una pedagogía integral, holística orientada hacia el desarrollo de competencias emocionales, que vaya más allá de los saberes cognitivos, sino que trascienda y de respuesta a las necesidades de los jóvenes de la postmodernidad, que impacte en diferentes áreas de la vida de las personas, como menciona Bisquerra y Pérez (2007) éstas influyen positivamente en los procesos de aprendizaje, las relaciones interpersonales, la solución de problemas, la consecución y mantenimiento de un puesto de trabajo, favoreciendo un desarrollo saludable e integral, promoviendo así el bienestar de los jóvenes.

Las competencias emocionales se basan en la inteligencia emocional, propuesta en sus comienzos por Salovey y Mayer (2004) y que años más tarde fue popularizada por Goleman (1999) con estudios más amplios y reconocidos a nivel internacional. Estas competencias integran elementos de un marco teórico más amplio, Bisquerra (2003) afirma en sus trabajos que "La educación emocional es una innovación educativa que responde a necesidades sociales no atendidas en las materias académicas ordinarias" (p.8)

La fundamentación está en el concepto de emoción, teorías de la emoción, la neurociencia, la psiconeuroinmunología, la teoría de las inteligencias múltiples, la inteligencia emocional, la educación psicológica, la educación para la salud, las habilidades sociales, entre otros." de igual forma el autor plantea que "El objetivo de la educación emocional es el desarrollo de competencias emocionales: conciencia emocional, regulación emocional, autogestión, inteligencia interpersonal, habilidades de vida y bienestar".

La educación emocional juega un papel fundamental en cada espacio de la vida de las personas, por cuanto concibe a éstas y desde un punto de vista holístico y preventivo, en la búsqueda de mejores estilos de vida y de relaciones intrapersonales e interpersonales más saludables. De este modo, se potencia el desarrollar habilidades, conocimientos y 
destrezas que promuevan la adaptación positiva, la conducta proactiva resiliente, regulación con consciencia emocional, autoeficacia para el afrontamiento de conflictos o dificultades familiares, laborales, comunitarias, entre otras, para el desarrollo humano con calidad de vida en espacios educativos incluyentes.

En el Estado Falcón, Venezuela, existe gran cantidad de jóvenes desescolarizados excluidos del sistema formal con escasas oportunidades de acceso a una educación de calidad para el desarrollo de competencias técnico profesionales y socioemocionales que faciliten su inserción en el mercado laboral. Muchos de ellos, con altos niveles de desesperanza aprendida producto del despegue fallido hacia la adolescencia sin redes de apoyo, con altos niveles de pobreza, ubicados en zonas urbanas marginales y áreas rurales, convirtiéndose en factores de riesgo psicosocial para estos jóvenes.

Para abordar esta problemática de carácter psicosocial emergen las instituciones promotoras del proceso los Centros de Capacitación Laboral (CECAL) asumiendo la problemática desde los diferentes campos de acción en las que vienen desarrollando sus propuestas educativas. A tal efecto, ha diseñado un currículo flexible en el que capacitación y educación básica se integran en función de una visualización global de la problemática laboral y el mejoramiento y desarrollo de la calidad de vida de las personas, el cual fue presentado y aprobado por el Ministerio del Poder Popular para la Educación, en donde se le otorga el reconocimiento y población que, por diferentes motivos, se encuentra excluido de la educación formal (AVEC, 1999).

Ahora bien, el Centro Educativo de Capacitación Laboral San Juan Bosco y la Red de Juventud Trabajo, perteneciente a la asociación civil de los Salesianos, ofrecen a los jóvenes que han quedado fuera del sistema escolar la oportunidad de realizar diferentes estudios, con la intención de ser insertados en el ámbito laboral mediante cursos certificados. El ideario o
Carácter Propio que define el Centro de Capacitación, es como decía Don Bosco formar "Buenos Cristianos y Honestos Ciudadanos", respetuosos con otras opciones educativas, y conscientes de la importancia que tiene el hecho de participar en la acción educativa.

Considerando entonces para abordar esta investigación como objetivo general: Determinar las competencias emocionales presentes en los jóvenes del Centro de Capacitación Laboral San Juan Bosco. Los objetivos específicos fueron: 1. Caracterizar los factores demográficos de los jóvenes del Centro de Capacitación Laboral San Juan Bosco. 2. Identificar las competencias emocionales intrapersonales presente en los jóvenes del Centro de Capacitación Laboral. 3. Identificar las competencias emocionales interpersonales presente en los jóvenes del Centro de Capacitación Laboral. 4. Comparar las competencias emocionales de los jóvenes escolarizados con los jóvenes desescolarizados del Centro de Capacitación Laboral San Juan Bosco. 5. Comparar el desarrollo de competencias emocionales con los factores demográficos de los jóvenes del Centro de Capacitación Laboral San Juan Bosco.

La investigación se justificó desde varios aspectos, entendiendo que el desarrollo de la competencia emocional, considerada ésta como habilidad básica para la vida, desemboca en la educación emocional, a través de una pedagogía afectiva sustentada en la neurociencia, psicología social, paradigma constructivista y la educación religiosa. Para ello teóricamente, se adoptaron los criterios de competencia emocional de Bisquerra (2003, 2007), centrada en la adquisición de conocimientos a otro enfoque orientado al desarrollo de competencias. Un tipo de competencias son las competencias emocionales.

\section{MÉTODO}

Dadas las características del estudio, se seleccionó la investigación descriptiva, con el objeto de llevar a la práctica modelos que 
permitan determinar las competencias emocionales en los jóvenes, Sabino (2007). De acuerdo con el tipo de investigación, el diseño de la misma es el no experimental, el cual Hernández et al. (2006), define como: aquel que se realiza sin manipular en forma deliberada ninguna variable.

En tal sentido se enmarcó conjuntamente en un diseño transeccional, el cual expone Hernández et al., (2006) como: recolectar datos en un solo momento, en un tiempo único. Su propósito es describir variables y analizar su incidencia e interrelación en un momento dado. Según lo citado, se procesó la información proveniente del instrumento que se aplicó en un momento único a la población en estudio.

La misma estuvo constituida por 273 jóvenes pertenecientes a los dieciséis (16) talleres que conforman el CECAL San Juan Bosco.

Tabla 1.Características de la Población

\begin{tabular}{ll}
\hline Participantes & Cantidad \\
\hline Escolarizados & 232 \\
No escolarizados & 41 \\
Total & 273 \\
\hline
\end{tabular}

Fuente: Elaboración propia.

Se empleó el muestreo de tipo no probabilístico, de carácter intencional, dado que la selección de los sujetos de investigación no dependió de que todos tuviesen la misma probabilidad de ser seleccionados, sino de la selección de los investigadores, a través de criterios con anticipación siendo estos necesarios para el logro de los objetivos. (Hernández et al., 2006).En este sentido, la muestra estuvo conformada por 59 jóvenes cursantes de los distintos talleres, distribuidos en escolarizados y no escolarizados que están en proceso de pasantías y que pronto van a egresar de la institución (Ver Tabla 2)

Tabla 2. Muestra representativa

\begin{tabular}{ll}
\hline Participantes & Cantidad \\
\hline Escolarizados & 44 \\
No escolarizados & 15 \\
Total & 59 \\
\hline
\end{tabular}

Fuente: Elaboración propia.

Para esta investigación se empleó como instrumento el Cuestionario de Desarrollo Emocional para Adultos (QDE-A). El QDE-A, que se enmarca en la línea de investigación sobre educación emocional del GROP (Grupo de Investigación en Orientación Psicopedagógica). Se trata de un cuestionario de autoinforme fundamentado en el marco teórico de la educación emocional desarrollado por el GROP (Bisquerra, 2007), según el cual la competencia emocional se compone de cinco dimensiones: conciencia emocional, regulación emocional, autonomía emocional, competencias sociales y competencias para la vida y el bienestar. La fiabilidad medida por el alfa de Cronbach es de 0,92, para la escala completa y superior a 0.70 para cada una de las dimensiones, lo que permite afirmar que este cuestionario es altamente confiable para evaluar el nivel de competencia emocional en adultos $\mathrm{y}$ 
fundamentar las intervenciones en educación emocional.

De acuerdo a las características de los jóvenes del Centro de Capacitación Laboral San Juan Bosco, se hizo necesario hacer una adaptación al instrumento planteado anteriormente, puesto que los jóvenes atendidos en dicha institución poseen edades entre 14 y 25 años;éstas varían en relación a las presentadas por los autores en el QDE-A, ya que comprenden un rango de mayor edad que las presentadas en el Centro de Capacitación; de igual modo también se hicieron las adaptaciones dado que los sujetos de investigación provienen de las zonas populares y áreas suburbanas, caracterizados por contar con muy pocos recursos económicos, culturales, académicos puesto que en algunos casos se encuentran desescolarizados y/o son desertores de la educación regular, es decir, la población y muestra objeto de estudio posee un rango de menor índice en comparación con los encuestados por los autores.

Por lo antes mencionado, se propusieron adaptaciones que consistieron en la modificación de la escala de Likert y el ajuste e inclusión en algunas proposiciones. Se diseñó como instrumento un cuestionario dirigido a los jóvenes participantes, conformado por 52 ítems, basándose el mismo en una escala tipo Likert con 5 alternativas de respuestas: totalmente en desacuerdo, en desacuerdo, indiferente, de acuerdo y totalmente en de acuerdo.

Tabla 3. Baremo para la interpretación de la Media

\begin{tabular}{clll}
\hline Grupo & \multicolumn{1}{c}{ Alternativas } & Valores & Categoría \\
\hline 5 & Totalmente de acuerdo & $4,21-5,00$ & Muy bueno \\
4 & De acuerdo & $3,41-4,20$ & Bueno \\
3 & Indiferente & $2,61-3,40$ & Regular \\
2 & En desacuerdo & $1,81-2,60$ & Deficiente \\
1 & Totalmente en desacuerdo & $1,00-1,80$ & Muy deficiente \\
\hline
\end{tabular}

Fuente: Elaboración propia.

Para constatar el grado de validez del instrumento y dadas las modificaciones hechas, se solicitó a tres (3) expertos en la materia, quienes verificaron: Pertinencia en los ítems, con relación los objetivos, a la variable en estudio e indicadores.

Con respecto a la confiabilidad de los instrumentos de recolección de datos se aplicó una prueba piloto. Para Tamayo y Tamayo (2003) esta permite ver las deficiencias existentes en torno al diseño metodológico y conduce a la realización de los ajustes necesarios e igualmente pondrá de manifiesto las ventajas y desventajas en torno a la investigación que se realizará posteriormente.

Así mismo, Hernández et al., (2006) plantean que la prueba piloto se aplica a personas con características semejantes a las de la muestra o población. Se realiza con una pequeña muestra (inferior a la definitiva).Los resultados de la prueba piloto fueron sometidos al cálculo del coeficiente de confiabilidad "Alpha de Cronbach", utilizado para medir la correspondencia de los objetivos con las variables e indicadores, demostrando así su nivel de aplicabilidad.

En este sentido, los resultados de las pruebas estadísticas para establecer el índice de confiabilidad determinó que el instrumento para la obtención de información referida a competencias emocionales de los jovenes del Centro de capacitación laboral San Juan Bosco, obtuvo un coeficiente Alfa Cronbach de 0,995, resultando ser muy altamente confiable; de la misma forma el método de partición por mitades arrojó finalmente un resultado de 0,991 a una parte y 0.990 a la segunda parte la cual reflejo ser muy alta en cuanto a la confiabilidad. 
RESULTADOS

Se destaca del primer objetivo de nuestra investigación que las edades más predominantes de los jóvenes del Centro de Capacitación abarca de los 17 años a los 19 años, en su mayoría del sexo masculino, de igual forma se observó que la mayoría es escolarizada, viven con sus padres, en casas propias, su núcleo familiar está completo y ellos son los mayores entre sus hermanos. Y finalmente la gran mayoría no posee empleo, por lo que buscan capacitarse en un oficio e ingresar en el ámbito laboral.

Todos los datos presentes en el perfil demográfico de los estudiantes escolarizado y no escolarizado del Centro de Capacitación Laboral "San Juan Bosco", enfatizaron el modelo de desarrollo positivo del adolescente de Oliva (2010). Para este objetivo implicó la promoción del bienestar emocional, relaciones interpersonales, bienestar material, desarrollo personal, bienestar físico, autodeterminación, inclusión social y derechos, para vivir plena y dignamente.

Con el segundo objetivo se logró constatar que un gran número de jóvenes están prestos a desarrollar en un nivel considerable conciencia emocional, es decir, son capaces de conocer sus propias emociones y las emociones de los demás; sin embargo, tanto para el indicador de regulación emocional como para el de autonomía emocional los niveles son regulares por lo que se deben reforzar aspectos importantes como la expresión emocional, habilidades de afrontamiento, así como autoestima, responsabilidad, automotivación, autoeficacia, actitud positiva, entre otros.

Por consiguiente los resultados de la dimensión competencias intrapersonales, evidencian que los estudiantes del Centro de Capacitación Laboral "San Juan Bosco", están inclinados hacia la consolidación de competencias emocionales que les permita analizar sus fortalezas y aprender de sus limitaciones y sobrepasarlas. A partir de Goleman (1999), existen doce actitudes que se mencionan a continuación: Conciencia emocional, Autoevaluación precisa, Confianza en uno mismo, Autocontrol, Confiabilidad, Escrupulosidad, Adaptabilidad, Innovación, Afán de triunfo, Compromiso, Iniciativa, Optimismo.

Ahora bien, sobre el tercer objetivo investigativo, los resultados de la dimensión competencias interpersonales, evidencian que los estudiantes del Centro de Capacitación Laboral "San Juan Bosco" están inclinados hacia el fortalecimiento de competencias sociales que faciliten la conducta prosocial con altos niveles de autoeficacia emocional o personal, indispensable en la inserción en el mercado laboral donde no sólo se exige el cumplimiento de objetivos de desempeño de tareas, sino a su vez habilidades de comunicación, afrontamiento de conflictos, trabajo en equipo, alto desempeño, respeto a la autoridad, entre otros.

Se observó un nivel bajo de $12,00 \%$ de rechazo o indiferencia $(10,62 \%)$, unido a otro $7,36 \%$ totalmente en desacuerdo ante la propuesta de la escala de Bisquerra (2003). Para Bandura (1984) existen escasos niveles de autoeficacia hacia la integración, socialización y adaptación; en otro orden menor o muy bajo reporta el 7,36\%.

Por consiguiente, los resultados de la dimensión competencias interpersonales evidencian que los estudiantes del Centro de Capacitación Laboral "San Juan Bosco" están inclinados hacia la consolidación de competencias sociales que faciliten la conducta prosocial con altos niveles de autoeficacia emocional o personal, indispensable en la inserción en el mercado laboral donde no sólo se exige el cumplimiento de objetivos de desempeño de tareas, sino a su vez habilidades de comunicación, afrontamiento de conflictos, trabajo en equipo, alto desempeño, respeto a la autoridad, entre otros.

En cuanto al cuarto objetivo del estudio se pudo comprobar una correlación altamente positiva y significativa medida con la escala de Bisquerra (2003), lo cual indica el peso que tiene 
el desarrollo de todas las competencias emocionales, unas en mayor rango como las habilidades de vida y bienestar y la conciencia emocional y otras, que aunque las demuestran de forma regular. Esta realidad coincide con los planteamientos de Bustamante (2002), en cuanto que las competencias entraron a la educación por influencia de factores externos tales como la competencia empresarial, la globalización y la internacionalización de la economía, con un bajo grado de estudio, análisis crítico y discusión por parte de la comunidad educativa.

Esta correlación positiva da cuenta además de los requerimientos de la tarea de la orientación educativa en el desarrollo de competencias educativas en el ciclo evolutivo de los adolescentes en transición hacia la vida adulta familiar, personal, social, profesional o laboral; siendo uno de los pilares fundamentales de esta disciplina procurar el desarrollo humano con calidad de vida.

Y finalmente en relación al quinto objetivo, se pudo constatar que escasamente existe relación entre los elementos configurativos de los factores sociodemográficos y el desarrollo de competencias emocionales de los sujetos de estudio.

Los resultados obtenidos con la comparación de los factores demográficos con respecto a las competencias emocionales es opuesta a los supuestos esperados en cuanto que los factores sociodemográficos de los sujetos estudiados, escasamente determinan el desarrollo de competencias emocionales para el control o manejo adecuado de las emociones, autonomía personal, conocimiento o respeto por sí mismo de la dimensión competencia intrapersonal; de igual forma, con respecto a las competencias sociales y de las competencias de vida y bienestar. Una posible explicación obedezca al hecho que los factores de riesgos y/o factores de protección en la adolescencia tardía están más bien asociados a los procesos de socialización donde interviene la familia, la escuela, la comunidad, las amistades o la sociedad en general, sin dejar de lado la influencia de los factores socioeconómicos en el comportamiento humano y la autoeficacia emocional.

\section{CONCLUSIONES}

Esta investigación consistió en la exposición de un modelo pentagonal de habilidades o competencias emocionales: conciencia emocional, regulación emocional, autonomía emocional, competencia social, habilidades de vida para el bienestar. A nivel educativo, es importante abordar la sustentación de dicho estudio, por cuanto permite determinar las competencias emocionales en los jóvenes del Centro de Capacitación Laboral San Juan Bosco a través de estrategias de educación emocional consustanciales a la formación laboral dentro de la perspectiva del desarrollo humano a nivel práctico.

A partir de esta investigación se pueden desarrollar y perfeccionar nuevas estrategias, donde se permita estimular las competencias emocionales de los educandos contribuyendo a su proceso de aprendizaje y su desarrollo como persona.

Se destaca en estas líneas finales que se encontró la presencia de la mayoría de competencias emocionales en los jóvenes del Centro de Capacitación Laboral, a pesar de vivir en condiciones de riesgo psicosocial, lo que podría inferirse que podría estar asociado al apoyo psicoeducativo que encuentran en el Centro de capacitación, el grupo de estudio, sus profesores como tutores integrales.

Además se comprueba una correlación altamente positiva y significativa medida con la escala de Bisquerra (2003), indicando el peso que tiene el desarrollo de todas las competencias emocionales, unas en mayor rango como las habilidades de vida y bienestar, así como la referida a la conciencia emocional y otras que, aunque de forma regular, están 
presentes en los jóvenes y pueden ser elementos de estudio para otras investigaciones.

\section{REFERENCIAS}

AVEC (1999). Diseño Curricular de los Centros Educativos de Capacitación Laboral (CECAL). Presentado al Ministerio del Poder Popular para la Educación por la Asociación Venezolana de Educación Católica AVEC. Caracas

Bandura, A. (1984). Teoría del Aprendizaje Social. Madrid: Editorial Espasa Calpe

Bisquerra, R. (2003). Educación Emocional y Competencias Básicas para la Vida. Revista de Investigación Educativa, Vol. 21, no 1. 743. Barcelona España

Bisquerra, R., y Pérez Escoda, N. (2007). Las competencias emocionales. Revista de
Educación siglo XXI, 10. 61-82. Barcelona, España

Bustamante, G. (2002). El concepto de competencias II: Una mirada interdisciplinar. Bogotá: Sociedad Colombiana de Pedagogía

Fondo de las Naciones Unidas para la Infancia. UNICEF. (2011). La Adolescencia. Una Época de Oportunidades. Nueva York, EEUU: Autor

Goleman, D. (1999).Inteligencia emocional, Barcelona: Kairós

Hernández S. R, Fernández, C. y Baptista, P. (2006) Metodología de la investigación. 5ta edición. México: Editores Mc Graw-Hill

Salovey, P., y Mayer, J. D. (2004). Emotional intelligence: key readings on the Mayer and Salovey model. New York: Dude Publishing. 